\title{
INFRARED AND OPTICAL SPECTROSCOPY OF ASTROPHYSICAL MOLECULES
}

\author{
PETER F. BERNATH \\ University of Waterloo, Department of Chemistry \\ Waterloo, Ontario, Canada N2L 3G1
}

\begin{abstract}
The recent laboratory spectroscopy of a number of astrophysically important molecules are discussed. Examples include small molecules such as $\mathrm{CrH}, \mathrm{HF}, \mathrm{FeF}, \mathrm{SiS}, \mathrm{CH}_{4}, \mathrm{H}_{2} \mathrm{O}, \mathrm{SiO}$ and $\mathrm{TiO}$ as well as large molecules such as $\mathrm{C}_{60}$ and polycyclic aromatic hydrocarbons (PAHs). Many of these examples illustrate the utility of infrared and far infrared emission spectroscopy. The close coupling of laboratory spectroscopy and molecular astronomy has led to advances in both areas.
\end{abstract}

\section{Introduction}

Molecules are found in a wide variety of astronomical objects including interstellar clouds, planetary nebulae, circumstellar envelopes, stellar atmospheres and comets. Molecular observations provide a great deal of useful information about physical properties and chemical composition. Molecular spectra provide diagnostics that probe the temperature, pressure and composition.

Although the first detections of molecules in extraterrestrial environments were carried out in the visible region, the majority of recent discoveries have come in the millimeter wave region. The use of sensitive array detectors has, however, revitalized visible spectroscopy and infrared array detectors are starting to have an impact in infrared astronomy. As infrared and submillimeter wave astronomy expands, molecules will play an increasingly important role in astrophysics.

In this paper, recent work in our laboratory on the near infrared, infrared and far infrared spectroscopy of astrophysical molecules is discussed. The electronic spectra of $\mathrm{CrH}$, $\mathrm{TiO}$ and $\mathrm{FeF}$ near $1 \mu \mathrm{m}$ were analyzed. Vibration-rotation emission spectra of $\mathrm{HF}, \mathrm{SiS}, \mathrm{SiO}, \mathrm{H}_{2} \mathrm{O}$ and $\mathrm{CH}_{4}$ were measured. For large molecules, the vibration-rotation bands of $\mathrm{C}_{60}, \mathrm{C}_{70}$ and three PAHs (naphthalene, pyrene and chrysene) were recorded in the gas 
phase. The laboratory measurements for $\mathrm{TiO}$ and $\mathrm{SiO}$ were combined with data from sunspot spectra to provide improved spectroscopic constants. The hot $\mathrm{H}_{2} \mathrm{O}$ emission spectra allowed the identification of $\mathrm{H}_{2} \mathrm{O}$ absorption lines in the infrared spectra of sunspots.

\section{Near-infrared electronic emission spectra}

The near infrared spectra of cool stars show strong absorption bands of metal hydrides and metal oxides. The $\mathrm{CrH}$ molecule has been identified in sunspots (Engvold et al. 1980) and in S stars (Lindgren \& Olofsson 1980) through the $\mathrm{A}^{6} \Sigma^{+}-\mathrm{X}^{6} \Sigma^{+}$transition near $861 \mathrm{~nm}$ (Kleman \& Uhler 1959).

We have recorded an improved spectrum of the $0-0$ band of the $\mathrm{A}^{6} \Sigma^{+}$ - $\mathrm{X}^{6} \Sigma^{+}$transition of $\mathrm{CrH}$ using a hollow lamp and a Fourier transform spectrometer (Ram et al. 1993). With these improved line positions, L. Wallace of Kitt Peak National Observatory has looked in a new sunspot spectrum (Ram et al. 1996c) and found no convincing evidence for the presence of $\mathrm{CrH}$. In addition to this near infrared data, Wallace has prepared new infrared sunspot and photospheric solar atlases (Wallace et al. 1996).

In the course of hunting for $\mathrm{CrH}$ lines in the sunspot spectra, new lines of $\mathrm{TiO}$ were identified. The $\delta$ system of $\mathrm{TiO}\left(\mathrm{b}^{1} \Pi-\mathrm{a}^{1} \Delta\right)$ has a $0-0$ band near $886 \mathrm{~nm}$. This transition is well known in the spectra of M stars (Kirkpatrick et al. 1991), but has not been previously noted in sunspots. In fact, the near infrared bands of $\mathrm{TiO}, \mathrm{VO}$ and $\mathrm{FeH}$ are used in the detailed classification of $\mathrm{M}$ stars.

We measured new laboratory hollow cathode spectra of the $0-0$ and 1-1 bands of the $\mathrm{b}^{1} \Pi-\mathrm{a}^{1} \Delta$ transition of TiO. The laboratory data and the sunspot lines were combined to provide improved molecular constants (Ram et al. 1996c). In addition to the $\delta$ system of $\mathrm{TiO}$, a few weak lines of the $\phi$ system $\left(b^{1} \Pi-d^{1} \Sigma^{+}\right)$were also identified in the sunspot spectra at $1.1 \mu \mathrm{m}$.

The surprising discovery of rotational transitions of $\mathrm{AlCl}, \mathrm{KCl}, \mathrm{NaCl}$ and AlF in the carbon-rich object IRC+10216 (Cernicharo \& Guélin 1987) suggests that metal halide molecules may be of astrophysical importance. Iron- containing compounds are particularly favourable for detection in stellar atmospheres because of the relatively high cosmic abundance of iron. The presence of the $\mathrm{F}^{4} \Delta-\mathrm{X}^{4} \Delta$ transition in sunspots and cool stellar spectra suggests that $\mathrm{FeF}$ might also be detectable in certain objects. Although ${ }^{19} \mathrm{~F}$ is a fragile nucleus, it has an enhanced abundance in AGB stars (Jorissen et al. 1992).

There is a strong correspondence between the spectra of transition metal monofluorides and monohydrides so that a near infrared ${ }^{4} \Delta-{ }^{4} \Delta$ transition of $\mathrm{FeF}$ is expected. We have recorded the $0-1,0-0,1-0$ and $2-0$ bands of 
the $\mathrm{g}^{4} \Delta-\mathrm{a}^{4} \Delta$ transition of $\mathrm{FeF}$ in the 9000 to $12000 \mathrm{~cm}^{-1}$ region (Ram et al. 1996b). The FeF molecule was made in a carbon tube furnace by reaction of liquid $\mathrm{Fe}$ with $\mathrm{CF}_{4}$ gas. Near infrared emission from the furnace was recorded with a Fourier transform spectrometer.

\section{Vibration-rotation emission spectroscopy}

The element fluorine is difficult to detect in extraterrestrial sources. The ${ }^{19} \mathrm{~F}$ nucleus is easily destroyed by nucleosynthetic reactions in stellar interiors so its abundance is low. The detection of fluorine is also difficult because most of the strong atomic lines, including the resonance lines, are found in the vacuum ultraviolet region.

In cooler sources the vibration-rotation transitions of HF near $2.5 \mu \mathrm{m}$ are the most convenient and reliable lines for $\mathrm{F}$ abundance determinations. Recently the HF vibration-rotation lines were used by Jorissen et al. (1992) to measure ${ }^{19} \mathrm{~F}$ abundances in a large sample of cool stars. They found that AGB stars have up to 30 times the solar abundance of F, suggesting that ${ }^{19} \mathrm{~F}$ is synthesized in He-burning shells.

Curiously there were no laboratory measurements for the highly-excited transitions of HF seen in stellar atmospheres. We measured the vibrationrotation as well as the pure rotational transitions of HF and DF at temperatures of up to $2600 \mathrm{~K}$ (Ram et al. 1996a) in a furnace. These emission spectra were recorded by accident in the course of our work on the infrared spectra of metal fluorides.

Vibration-rotation emission spectroscopy is a very powerful technique for measuring the spectra of molecules at high temperatures. Traditionally infrared spectra have been recorded in absorption and the sensitivity advantage of laboratory emission spectroscopy at long wavelengths has not been appreciated. I have written a review article (Bernath 1996) on the virtues of the infrared emission technique.

The 1-0 vibration-rotation lines of SiS have been detected in absorption in IRC+10216 (Boyle et al. 1994). We recorded the vibration-rotation emission spectrum of SiS at $13 \mu \mathrm{m}$. The SiS molecules were made at 1300 $\mathrm{K}$ by the reaction of $\mathrm{SiS}_{2}$ solid with $\mathrm{Si}$ powder in a tube furnace (Frum et al. 1990). Comparison of the laboratory and astronomical infrared spectra confirmed the SiS identification in IRC+10216.

The original discovery and preparation of the $\mathrm{C}_{60}$ molecule (Kroto et al. 1985, Krätschmer et al. 1990) was motivated by astronomical considerations. Now that laboratory spectra of $\mathrm{C}_{60}$ are available, it is interesting to carry out an astronomical search for $\mathrm{C}_{60}$. Although the infrared spectra of solid $\mathrm{C}_{60}$ are well known, the low temperature gas phase spectra are not available. We, therefore, recorded the infrared emission spectra of gaseous 
$\mathrm{C}_{60}$ and $\mathrm{C}_{70}$ as a function of temperature and extrapolated the band positions to low temperatures (Nemes et al. 1994). For large molecules such as $\mathrm{C}_{60}$ excellent infrared emission spectra can be recorded, although the rotational structure is not resolved.

The strong $\mathrm{C}_{60}$ band at $1191 \mathrm{~cm}^{-1}$ is suitable for an astronomical search. We were unable to detect this band in IRC+10216 (Hinkle \& Bernath 1994) at Kitt Peak. Other pure carbon molecules such as $\mathrm{C}_{3}$ (Hinkle et al. 1988) and $\mathrm{C}_{5}$ (Bernath et al. 1989) have been identified in IRC+10216 by infrared spectroscopy at $5 \mu \mathrm{m}$. However the presence of large amounts of $\mathrm{H}_{2}$ in this object may inhibit the chemical production of $\mathrm{C}_{60}$. More favourable sources for $\mathrm{C}_{60}$ production may be $\mathrm{R}$ Coronae Borealis ( $\mathrm{R} \mathrm{CrB}$ ) stars that are carbon rich but hydrogen deficient. However, a search for $\mathrm{C}_{60}$ in three $\mathrm{R} \mathrm{CrB}$ stars with a cryogenic echelle spectrograph also proved negative (Clayton et al. 1995).

The Sun and, particularly, sunspots are rich sources of astronomical spectra of molecules. For example in the $10 \mu \mathrm{m}$ spectrum of a sunspot there is a dense forest of lines which can be assigned to SiO. In our laboratory a new $\mathrm{SiO}$ vibration-rotation emission spectrum was recorded by accident (Campbell et al. 1993). These laboratory measurements of $\mathrm{SiO}$ were combined with the sunspot data to provide a very extensive new set of line positions and molecular constants (Campbell et al. 1995).

In the infrared sunspot atlas (Wallace et al. 1996) numerous unidentified lines are present throughout the infrared region. We suspected that these lines were due to hot $\mathrm{H}_{2} \mathrm{O}$ but the available laboratory data were inadequate to confirm this. We, therefore, recorded new laboratory emission spectra of hot water at $1800 \mathrm{~K}$ in the infrared region. Comparison of the laboratory emission spectra of $\mathrm{H}_{2} \mathrm{O}$ and the sunspot absorption spectra identified most of the unassigned sunspot lines as $\mathrm{H}_{2} \mathrm{O}$ lines (Wallace et al. 1995).

Only a small fraction of the new water lines could be assigned quantum numbers. The water molecule is so light that the traditional power series expressions for the rotational energy levels are divergent. This massive breakdown of perturbation theory makes the assignment of quantum numbers to the lines very difficult. We have, therefore, begun a collaboration with the theoreticians O. Polyansky and J. Tennyson to apply more sophisticated approaches to the assignment of our spectra. Some preliminary assignments have already been made (Polyansky et al. 1996) and more work is in progress.

The infrared and far infrared spectrum of hot water is of enormous importance in molecular astronomy. Water is the main source of infrared opacity in all oxygen-rich cool objects. It is found in M stars and is particularly prominent in Mira variables (Hinkle \& Barnes 1979). New results from the ISO satellite are showing widespread water absorption and emission in 
many objects. One result of our work will be improved infrared opacities (Allard et al. 1994) for modelling cool stellar atmospheres.

In the spectra of substellar objects such as brown dwarfs like Gl 229B the infrared spectra of both $\mathrm{H}_{2} \mathrm{O}$ and $\mathrm{CH}_{4}$ are present (Oppenheimer et al. 1995). We have also recorded a new spectrum of hot $\mathrm{CH}_{4}$ in the $3 \mu \mathrm{m}$ region at the request of $\mathrm{T}$. Oka in order to assign lines detected in the impact of comet Shoemaker-Levy with Jupiter.

The infrared spectra of planetary nebulae such as NGC 7027 and H II regions show unidentified infrared emission bands (UIRs) at $3.3 \mu \mathrm{m}, 6.2 \mu \mathrm{m}$, $7.7 \mu \mathrm{m}, 8.7 \mu \mathrm{m}$ and $11.3 \mu \mathrm{m}$. The PAH hypothesis (Allamandola et al. 1989) attributes these bands to infrared emission from PAH molecules excited by strong UV radiation. These PAHs may be in the form of a gaseous, partlyionized, partly-dehydrogenated mixture of species. The PAH hypothesis was based on the similarity between the characteristic infrared absorption bands of solid PAH molecules in $\mathrm{KBr}$ pellets and the UIRs. A more appropriate comparison would be between the emission spectra of gaseous $\mathrm{PAH}$ molecules and the UIRs.

Kurtz (1992) and Joblin et al. (1995) have recorded some PAH emission spectra and we have extended these measurements into the far infrared region (Zhang et al. 1996). We find that even in the far infrared region excellent emission spectra can be recorded for napthalene, pyrene and chrysene. These experimental measurements are in excellent agreement with the ab initio predictions of Langhoff (1996). In contrast to the mid-infrared spectra of PAH molecules, the far infrared spectra are unique for each $\mathrm{PAH}$ molecule. In fact the far infrared region provides a "spectroscopic fingerprint" for each of the different PAH molecules.

\section{References}

Allamandola, L.J., Tielens, A.G.G.M., Barker, J.R. 1989, A\&AS 71, 733

Allard, F., Hauschildt, P.H., Miller, S., Tennyson, J. 1994, ApJ 426, L39

Bernath, P.F., Hinkle, K.H., Keady, J.J. 1989, Science 244, 562

Bernath, P.F. 1996, Chem. Soc. Rev. 25, 111

Boyle, R.J., Keady, J.J., Jennings, D.E., Hirsch, K.L., Wiedemann, G.R. 1994, ApJ 420, 863

Campbell, J.M., Dulick, M., Klapstein, D., White, J.B., Bernath, P.F. 1993, J. Chem. Phys. 99, 8379

Campbell, J.M., Klapstein, D., Dulick, M., Bernath, P.F., Wallace, L. 1995, ApJS 101, 237

Cernicharo, J., Guélin, M. 1987, A\&A 183, L10

Clayton, G.C., Kelly, D.M., Lacy, J.H., Little-Marenin, I.R., Feldman, P.A., Bernath, P.F. 1995, Astron. J. 109, 2096

Engvold, O., Wöhl, H., Brault, J.W. 1980. A\&AS 42, 209

Frum, C.I., Engleman, R., Bernath, P.F. 1990, J. Chem. Phys. 93, 5457

Hinkle, K.H., Barnes, T.G. 1979, ApJ 227, 923

Hinkle, K.H., Keady, J.J., Bernath, P.F. 1988, Science 241, 1319 
Hinkle, K.H., Bernath, P.F. 1994, unpublished results

Joblin, C., d'Hendecourt, L., Léger, A., Défourneau, D. 1995, A\&A 281, 923

Jorissen, A., Smith, V.V., Lambert, D.L. 1992, A\&A 261, 164

Kirkpatrick, J.D., Henry, T.J., McCarthy, D.W. 1991, ApJS 77, 417

Kleman, B., Uhler, U. 1959, Can. J. Phys. 37, 537

Krätschmer, W., Lamb, L.D., Fostiropoulos, K., Huffman, D.R. 1990, Nature 347, 354

Kroto, H.W., Heath, J.R., O'Brien, S.C., Curl, R.F., Smalley, R.E. 1985, Nature 318, 162

Kurtz, J. 1992, A\&A 255, L1

Langhoff, S.R. 1996, J. Phys. Chem. 100, 2819

Lindgren, B., Olofsson, G.S. 1980, A\&A 84, 300

Nemes, L., Ram, R.S., Bernath, P.F., Tinker, F.A., Zumwalt, M.C., Lamb, L.D., Huffman, D.R. 1994, Chem. Phys. Lett. 218, 295

Oppenheimer, B.R., Kulkarni, S.R., Mathews, K., Nakajima, T. 1995, Science 270, 1478

Polyansky, O.L., Busler, J.R., Guo, B., Zhang, K., Bernath, P.F. 1996, J. Mol. Spectrosc. 176,305

Ram, R.S., Jarman, C.N., Bernath, P.F. 1993, J. Mol. Spectrosc. 161, 445

Ram, R.S., Morbi, Z., Guo, B., Zhang, K., Bernath, P.F., Vander Auwera, J., Johns, J.W.C., Davis, S.P. 1996a, ApJS 103, 247

Ram, R.S., Bernath, P.F., Davis, S.P. 1996b, J. Mol. Spectrosc. 179, 282

Ram, R.S., Bernath, P.F., Wallace, L. 1996c, ApJS in press

Wallace, L., Bernath, P.F., Livingston, W., Hinkle, K., Busler, J., Guo, B., Zhang, K. 1995, Science 268, 1155

Wallace, L., Livingston, W., Hinkle, K., Bernath, P. 1996, ApJS in press

Zhang, K., Guo, B., Colarusso, P., Bernath, P.F. 1996, Science 274, 582

\section{Discussion}

Pecker: The ro-vibrational $T$ determined from $\mathrm{SiO}$, and perhaps $\mathrm{H}_{2} \mathrm{O}$, as observed in sunspots, could display a contradiction: these apparent contradictions may possibly be linked with (a) a Non-LTE situation (b) an inhomogeneous situation. As they are strongly $T$ dependent, these lines could be used for the determination of fine-structure characteristics both in the photosphere and in sunspots.

Bernath:: Yes, this is quite possible but we have not done it yet. Our temperature of $3200 \mathrm{~K}$ was determined from the $\mathrm{SiO}$ line intensities. We assume that the $\mathrm{H}_{2} \mathrm{O}$ is at the same temperature but we have not completed our analysis yet.

Langhoff: Both theoretical and experimental studies have shown that the probable carriers of the UIR bands are the PAH cations. Can you measure the FIR specra of PAH cations with this method?

Bernath: Yes, in principle it is possible but the measurement would be very difficult in the gas phase.

d'Hendecourt: FIR spectra of many $(\sim 50)$ PAHs have been measured (Mouton et al. 1996, A\&A 310, 297) in our lab. The observed transitions are strongly related to the geometry of the molecule. Thus, contrary to the $-\mathrm{CH}$ stretch $(3.28 \mu \mathrm{m})$, which is generic to a family of molecules, the FIR will display too many bands to be useful for detection. This may be similar to what happens in the visible where up to now, no specific PAH has been identified in spite of the presence of a few hundred DIBs. Bernath: Yes, there are many characteristic bands in the far infrared region. The hope is that there may be some sources in which only a few PAHs dominate in the mixture. In this case it should be possible to identify specific PAHs by matching with FIR laboratory spectra in the gas phase. 